\title{
Holographic Dark Energy: its Observational Constraints and Theoretical Features
}

\author{
Yin-Zhe Ma \\ Institute of Astronomy, University of Cambridge, Madingley Road, Cambridge, CB3 OHA, U.K.
}

\begin{abstract}
We investigate the observational signatures of the holographic dark energy model in this paper, including both the original model and a model with an interaction term between the dark energy and dark matter. We first delineate the dynamical behavior of such models, especially whether they would have a "Big Rip" for different parameters, then we use several recent observational data to give more reliable and tighter constraints on the models. The results favor the equation of state of dark energy crossing -1 , and the universe ends in the "Big Rip" phase. By using the Bayesian evidence as a model selection criterion to make the model comparison, we find that the holographic dark energy models are mildly favored by the observations compared with the $\Lambda \mathrm{CDM}$ model.
\end{abstract}

Keywords: holographic principle, interacting dark energy, observational constraints

PACS: 98.80.-k, 95.36.+x

\section{INTRODUCTION}

The nature of dark matter (DM) and dark energy (DE), which constitute about $95 \%$ of the total cosmic density, are among the most important problems in modern physics and astronomy [1]. Dark energy may be a problem that has to be solved in the framework of fundamental microscopic physics such as string theory [2].

Here we consider the dark matter-dark energy interaction in the case of the so-called holographic dark energy (HDE) model [3, 4, 5, 6, 7]. This is based on the holographic principle [8], which was inspired by the Bekenstein entropy bound of black holes [9]. If the quantum zero-point energy density $\rho_{\Lambda}$ is relevant to an ultraviolet cut-off, the total energy of the whole system with size $L$ should not exceed the mass of a black hole of the same size, thus we have $L^{3} \rho_{\Lambda} \leq L M_{\mathrm{pl}}^{2}$. The largest infrared cut-off $L$ is chosen by saturating the inequality so that the HDE density is

$$
\rho_{\mathrm{de}}=3 c^{2} M_{\mathrm{pl}}^{2} L^{-2},
$$

where $c$ is a numerical constant, and $M_{\mathrm{pl}} \equiv 1 / \sqrt{8 \pi G}$ is the reduced Planck mass. $L$ should be the size of the future event horizon, since only this case would result in a dark energy component which drives the accelerated expansion of the universe [6], i.e.

$$
L=R_{e h}(t)=a(t) \int_{a}^{\infty} \frac{d a^{\prime}}{H^{\prime} a^{\prime 2}} .
$$

If we consider the dark matter and dark energy interaction, the energy conservation equation will be written as

$$
\dot{\rho}_{m}+3 H \rho_{m}=Q
$$



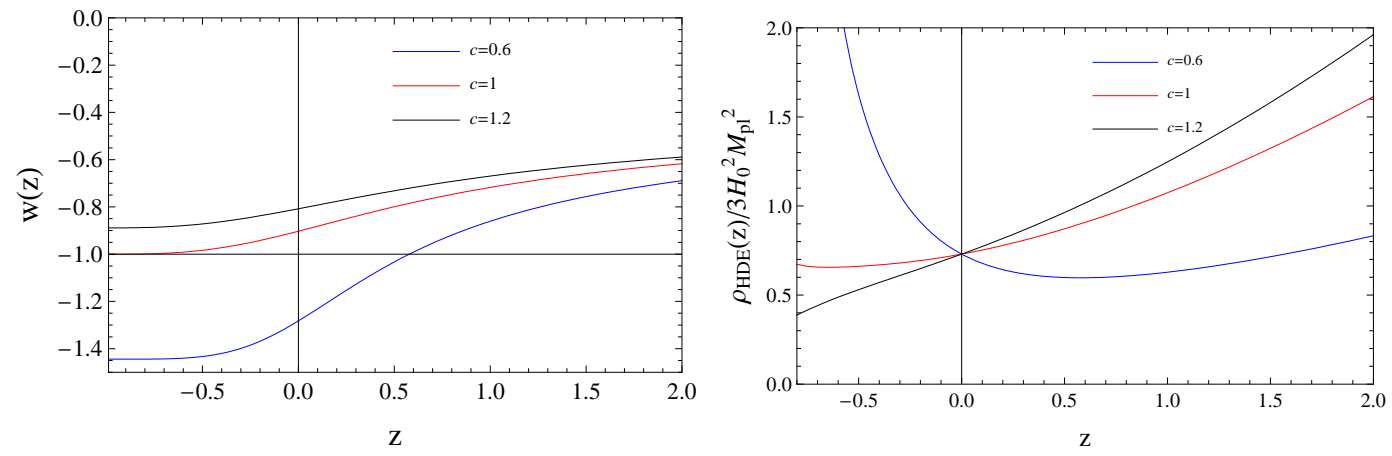

FIGURE 1. EEoS and HDE density in the holographic dark energy model for different value of $c$. Left: EEoS; Right: HDE density. Here we set $\Omega_{m 0}=0.27$.

$$
\dot{\rho}_{\mathrm{de}}+3 H\left(1+w_{\mathrm{de}}\right) \rho_{\mathrm{de}}=-Q,
$$

where $\rho_{m}, \rho_{\mathrm{de}}$ and $w_{\mathrm{de}}$ are matter density, HDE density and HDE equation of state respectively. Here we consider the physically plausible interaction as $Q=3 \alpha H \rho_{\mathrm{de}}(\alpha$ is a parameter describing the strength of interaction), which has been widely used for the interaction between the matter and massive scalar field [10]. Therefore, by combining the above equations and Friedmann equation, we quickly obtain the following equations which describe the evolution of the fractional energy density $\Omega_{\text {de }}$, Hubble parameter $H(z)$, and also the HDE effective equation of state (EEoS) (see [7] for details):

$$
\begin{gathered}
\frac{d \Omega_{\mathrm{de}}(z)}{d z}+\frac{\Omega_{\mathrm{de}}}{1+z}\left[\left(1-\Omega_{\mathrm{de}}\right)\left(1+\frac{2}{c} \sqrt{\Omega_{\mathrm{de}}}\right)-3 \alpha \Omega_{\mathrm{de}}\right]=0, \\
\frac{d H}{d z}=-\frac{H(z)}{1+z}\left[\frac{1}{2} \Omega_{\mathrm{de}}\left(1+3 \alpha+\frac{2}{c} \sqrt{\Omega_{\mathrm{de}}}\right)-\frac{3}{2}\right], \\
w_{\mathrm{eff}}(z)=w_{d e}+\alpha=-\frac{1}{3}-\frac{2}{3} \frac{\sqrt{\Omega_{\mathrm{de}}}}{c} .
\end{gathered}
$$

By solving the Eqs. (5), (6), and (7), we can know the dynamics of the HDE model as follows: For the non-interacting HDE model, i.e. $\alpha=0$, the evolution of HDE density and its EEoS will be affected by the value of parameter $c$ as shown in Fig. 1. If $c=1$, the EEoS of HDE will turn out to be -1 (Cosmological Constant), and the universe will end up in a de-Sitter phase. If $c>1$, the EEoS is always greater than -1 , and the HDE is just like the quintessence dark energy. On the contrary, if $c<1$, the EEoS will cross -1 at some time, and the universe will end up in a "Big Rip", indicating the HDE resembles the behavior of phantom dark energy [11].

For the interacting HDE model (IHDE), we vary the value of $\alpha$ but fix $c=1$ to see the interacting effect. In Fig. 2, we see that if $\alpha<0$, the dark matter "decays" into IHDE (Eqs. (3) and (4)), which will make the HDE density increase rapidly at the later era of cosmic evolution. Thus, effectively the IHDE will resemble the phantom dark energy with EEoS less than -1 in the near future (see the left of Fig. 2). On the other hand, if IHDE "decays" into dark matter, i.e. $\alpha>0$, the IHDE density would decay more rapidly, making the IHDE behave like a quintessence field. 

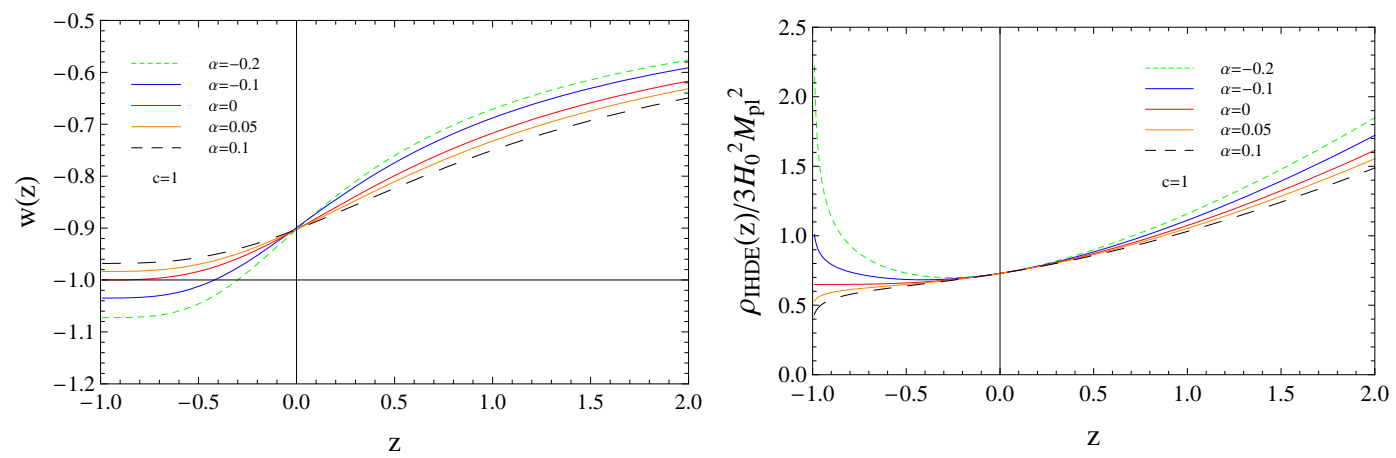

FIGURE 2. EEoS and IHDE density in the interacting holographic dark energy model for different value of $\alpha$. Left: EEoS; Right: HDE density. Here we set $\Omega_{m 0}=0.27$.

However, we want to ask the inverse questions: what values of parameter $c$ and $\alpha$ are preferred by the current observational data? In addition, which of these dynamic dark energy models are favored by current observational data compared with the concordance $\Lambda \mathrm{CDM}$ model?

\section{METHODOLOGY}

We utilize several data sets to constrain the parameters of the HDE and IHDE model, including the 182 high-quality type Ia supernovae [12], the baryon acoustic oscillation measurement from the Sloan Digital Sky Survey [13], 42 latest X-ray gas mass fraction data from Chandra observations [14], 27 GRB samples generated with $E_{\text {peak }}-$ $E_{\gamma}$ correlation [15], and the CMB shift parameter from the WMAP 3 year results [16]. To break the degeneracy and explore the power and differences of the constraints for these data sets, we use them in several combinations to perform our fitting: $\mathrm{SN}_{\text {sel }}+\mathrm{BAO}, \mathrm{SN}_{\mathrm{sel}}+\mathrm{BAO}+\mathrm{f}_{\text {gas }}$, and $\mathrm{SN}_{\text {sel }}+\mathrm{BAO}+\mathrm{f}_{\text {gas }}+\mathrm{GRB}+\mathrm{CMB}$.

For comparing different models, one must choose a statistical variable. The $\chi_{\min }^{2}$ is the simplest one and is widely used. However, for models with different numbers of parameters, the comparison using $\chi^{2}$ may not be fair, as one would expect that models with more parameters tend to have lower $\chi^{2}$. Instead, we use the Bayesian evidence (BE) as a model selection criterion. The Bayesian evidence of a model $M$ takes the form

$$
\mathrm{BE}=\int \mathscr{L}(\mathbf{d} \mid \theta, M) \mathbf{p}(\theta \mid M) d \theta
$$

where $\mathscr{L}(\mathbf{d} \mid \theta, M)$ is the likelihood function given the model $M$ and parameters $\theta$, and $\mathbf{p}(\theta \mid M)$ is the priors of the parameters. The BE may be the best model selection criterion, as it is the average of likelihood of a model over its prior of the parameter space and automatically includes the penalties of the number of parameters and data, so it is more direct, reasonable and unambiguous than the $\chi_{\min }^{2}$ model selection [17]. The logarithm of $\mathrm{BE}$ can be used as a guide for model comparison, and we choose the $\Lambda \mathrm{CDM}$ as the reference model: $\Delta \ln \mathrm{BE}=\ln \mathrm{BE}_{\text {model }}-\ln \mathrm{BE}_{\Lambda C D M}$. The strength of the evidence for the model is considered according to the numerical value of $\mathrm{BE}: \Delta \ln \mathrm{BE}<1$ (Weak); 
$1<\Delta \ln \mathrm{BE}<2.5$ (Significant) $; 2.5<\Delta \ln \mathrm{BE}<5$ (Strong to very strong); $\Delta \ln \mathrm{BE}>5$ (Decisive). We use the nested sampling technique to compute BE $[18,19]$.

\section{RESULTS}

In Table 1, we give the best fits and the $1 \sigma \mathrm{CL}$ of the HDE model parameters, as well as the value of $\ln \Delta \mathrm{BE}$ for the three data set combinations. The best fit of $c$ varies slightly across the different data sets, it is 0.761 for the $\mathrm{SN}+\mathrm{BAO}$ data set, but decreases slightly when the $f_{\text {gas }}$, GRB and CMB data are included. However, for all data sets, we have $c<1$ at more than $1.5 \sigma$, indicating that the EEoS of HDE will cross -1 at some redshift, and the energy density of HDE will diverge in the future (see the blue curve in Fig. 1). The

TABLE 1. The fitting result for the HDE model.

\begin{tabular}{c|c|c|c} 
& $\mathrm{SN}+\mathrm{BAO}$ & $\mathrm{SN}+\mathrm{BAO}+\mathrm{f}_{\mathrm{gas}}$ & $\mathrm{SN}+\mathrm{BAO}+\mathrm{f}_{\mathrm{gas}}+\mathrm{GRB}+\mathrm{CMB}$ \\
\hline$\Omega_{m 0}$ & $0.273_{-0.020}^{+0.020}$ & $0.270_{-0.018}^{+0.021}$ & $0.276_{-0.016}^{+0.017}$ \\
$c$ & $0.761_{-0.117}^{+0.154}$ & $0.745_{-0.101}^{+0.130}$ & $0.748_{-0.093}^{+0.108}$ \\
$\Delta \ln \mathrm{BE}$ & $0.09 \pm 0.12$ & $0.63 \pm 0.18$ & $0.65 \pm 0.18$
\end{tabular}

HDE model fits about equally well (ln $\mathrm{BE}=0.09$ ) as the $\Lambda \mathrm{CDM}$ when we only use the SNIa and BAO data. With $f_{\text {gas }}$, GRB and CMB data added, it fits mildly better than the $\Lambda \mathrm{CDM}$, but with the data presently available the difference is not significant (ln $\mathrm{BE}$ $=0.63 \sim 0.65$ ).

In Table 2, we show the fitting results for the IHDE model. From the best fits

TABLE 2. The Fitting results the IHDE model.

\begin{tabular}{c|c|c|c} 
& $\mathrm{SN}+\mathrm{BAO}$ & $\mathrm{SN}+\mathrm{BAO}+\mathrm{f}_{\mathrm{gas}}$ & $\mathrm{SN}+\mathrm{BAO}+\mathrm{f}_{\mathrm{gas}}+\mathrm{GRB}+\mathrm{CMB}$ \\
\hline$\Omega_{m 0}$ & $0.272_{-0.022}^{+0.023}$ & $0.275_{-0.021}^{+0.021}$ & $0.281_{-0.017}^{+0.017}$ \\
$\mathrm{c}$ & $0.592_{-0.113}^{+0.204}$ & $0.667_{-0.164}^{+0.321}$ & $0.692_{-0.107}^{+0.135}$ \\
$\alpha$ & $-0.020_{-0.145}^{+0.145}$ & $0.068_{-0.120}^{+0.093}$ & $-0.006_{-0.024}^{+0.021}$ \\
$\Delta \ln \mathrm{BE}$ & $0.41 \pm 0.12$ & $0.70 \pm 0.18$ & $0.75 \pm 0.18$
\end{tabular}

and $1 \sigma \mathrm{CL}$, the fractional energy density of dark matter is still around 0.27 , and the parameter $c$ is always less than 1 . The difference from the HDE model is that IHDE has the interacting parameter $\alpha$ here and its value is either positive or negative, but
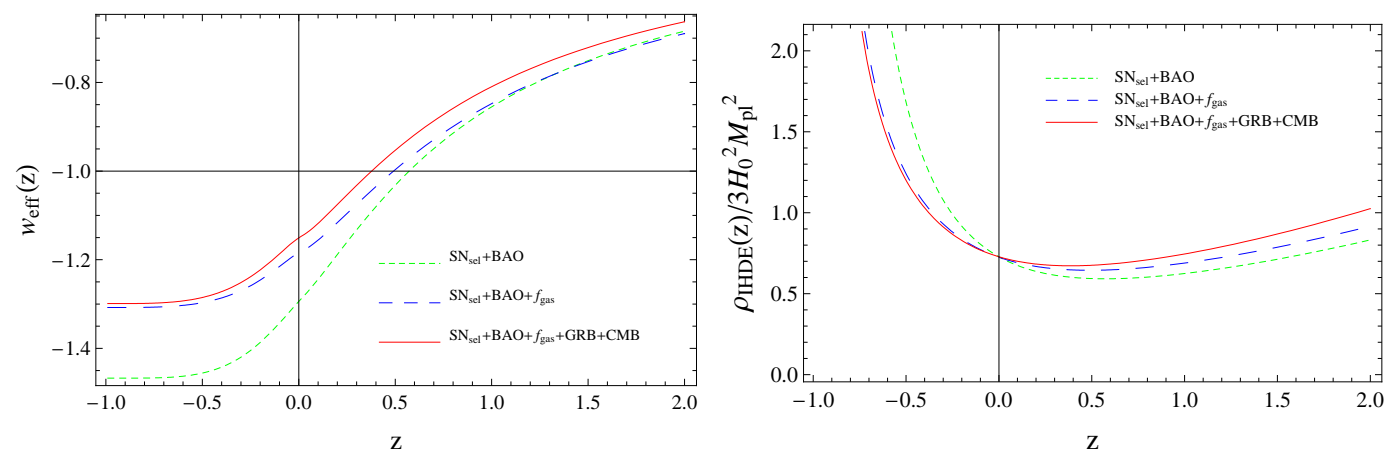

FIGURE 3. EEoS and energy density evolution for the best fit IHDE models. 


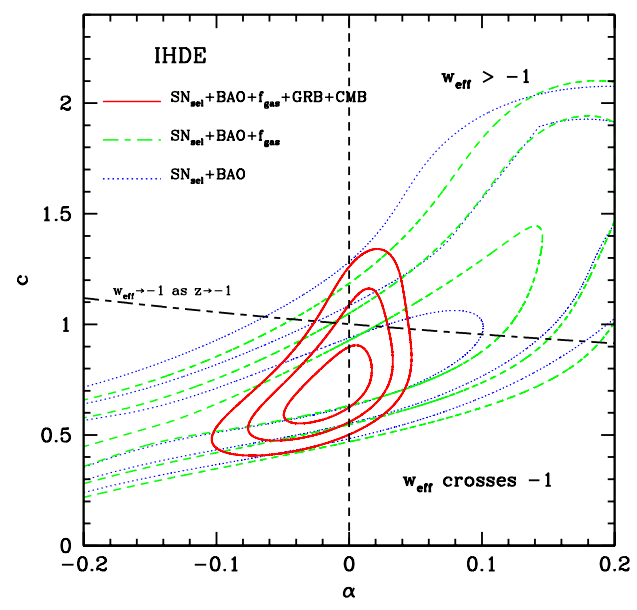

FIGURE 4. The contours of $\mathrm{c}$ vs. $\alpha$ in the IHDE model.

always very small and around zero. If you look closer at it, zero is always covered in the $1 \sigma \mathrm{CL}$, indicating that there is little evidence for the interaction. Furthermore, the Bayesian Evidence is always positive but less than 1, if you compare them with the previous Bayesian Evidence of non-interacting case, you can see that the number is slightly greater than the previous case, which means that the IHDE model is a little more favored than the HDE model but the evidence is still not strong.

Using the best fitting values for the three data sets, we also plot the effective equation of state and dark energy density in Fig. 3. We can see that all three data sets suggest the equation of state crosses -1 and the universe ends in the "Big Rip" in the future, just like the HDE model.

The contour map for $c$ and $\alpha$ is plotted in Fig. 4. In Fig. 4, the contours corresponding to the data sets $\mathrm{SN}+\mathrm{BAO}+f_{\text {gas }}+\mathrm{GRB}+\mathrm{CMB}$ are much tighter than the other data sets. This is because we use the data set GRB and CMB, which has very large redshift distribution so they break the degeneracy of the parameter $c$ and $\alpha$. We also mark the $\alpha-c$ values for which $w_{e f f}=-1$ as a dashed line. This forms the dividing line between quintessence-like and phantom-like behavior. For the best fit parameters of all three data set combinations, $c \sim 0.6$, so $c<1$. The value of $\alpha$ varies more, but all consistent with being 0 within $1.5 \sigma$, which suggests the evidence for the interaction is very weak. In any case, for all three data set combinations, the best fit value resides in the phantom-like region, although a large area of quintessence-like region is also allowed.

\section{CONCLUSION}

In this paper we first introduced the holographic dark energy model with the interacting term $Q=3 \alpha H \rho_{\mathrm{de}}$, so the non-interacting case could be viewed as the special case with $\alpha=0$. We illustrated the dynamical behavior of these models by choosing some representative values of the parameters $c$ and $\alpha$. The condition for the model to have a "Big Rip" is determined. Second, we utilize several data sets from the recent observations to constrain the models. The best-fits for the three data sets are given in Table 
1 for the HDE model, and Table 2 for the IHDE model. For both the HDE and IHDE models, the data favors "phantom" behavior slightly, i.e. the dark energy initially has $w_{\text {eff }}>-1$, but eventually crossing the phantom dividing line, and the model ends with a "Big Rip". However, quintessence-like behavior is also allowed with the present data. Next, we utilize the Bayesian evidence (BE) as a model selection criterion to compare the holographic models with the $\Lambda$ CDM model for the three data sets. Both the HDE and the IHDE model are mildly favored by the current observational data set, although the evidence is weak.

In brief, we conclude that according to the observational data, the holographic dark energy model, especially the interacting holographic dark energy model is mildly favored by the current data, and for the best fit model the equation of state for both the HDE and IHDE crosses -1, for which the Universe ends up in a "Big Rip".

\section{ACKNOWLEDGMENTS}

I acknowledge the support from the Cambridge Overseas Trust and a studentship from Trinity College, Cambridge, as well as the National Science Foundation in China. I also thank Xuelei Chen, Yan Gong and many other colleagues for the helpful discussions, and David Cline for inviting me to give this talk.

\section{REFERENCES}

1. P. J. Steinhardt, in Critical Problems in Physics, edited by V. L. Fitch and D. R. Marlow (Princeton University Press, Princeton, NJ, 1997).

2. E. Witten, hep-ph/0002297.

3. A. G. Cohen, D. B. Kaplan and A. E. Nelson, Phys. Rev. Lett. 82, 4971 (1999) [hep-th/9803132].

4. P. Horava and D. Minic, Phys. Rev. Lett. 85, 1610 (2000) [hep-th/0001145]; S. D. Thomas, Phys. Rev. Lett. 89, 081301 (2002).

5. S. D. H. Hsu, Phys. Lett. B 594, 13 (2004) [hep-th/0403052].

6. M. Li, Phys. Lett. B 603, 1 (2004) [hep-th/0403127].

7. Y. Z. Ma, Y. Gong and X. Chen, 0711.1641, [astro-ph], accepted for publication in EPJC.

8. G. 't Hooft, gr-qc/9310026; L. Susskind, J. Math. Phys. 36, 6377 (1995) [hep-th/9409089].

9. J. D. Bekenstein, Phys. Rev. D 7, 2333 (1973); J. D. Bekenstein, Phys. Rev. D 9, 3292 (1974); J. D. Bekenstein, Phys. Rev. D 23, 287 (1981); J. D. Bekenstein, Phys. Rev. D 49, 1912 (1994); S. W. Hawking, Commun. Math. Phys. 43, 199 (1975); S. W. Hawking, Phys. Rev. D 13, 191 (1976).

10. P. G. Ferreira and M. Joyce, Phys. Rev. D 58, 023503 (1998) [astro-ph/9711102]; A. Berera and L. Z. Fang, Phys. Rev. Lett. 74, 1912 (1995) [astro-ph/9501024].

11. R. R. Caldwell, Phys. Lett. B 45, 23 (2002), [astro-ph/9908168].

12. A. G. Riess et al., Astrophys. J. 656, (2007) [astro-ph/0611572]; P. Astier et al., Astron. Astrophys. 447, (2006) [astro-ph/0510447]; W. M. Wood-Vasey et al., (2007) [astro-ph/0701041]; S. Nesseris and L. Perivolaropoulos, JCAP. 0702, 025 (2007) [astro-ph/0612653.]

13. D. J. Eisenstein et al., Astrophys. J. 633, 560-574 (2005) [astro-ph/0501171]; D. N. Spergel et al., Astrophys. J. Suppl. Ser. 170, 377 (2007) [astro-ph/0601133].

14. S. W. Allen et al. Mon. Not. Roy. Astron. Soc. 383, 879 (2008), 0706.0033, [astro-ph].

15. B. E. Scheafer, Astrophys. J. 660, 16 (2007) [astro-ph/0612285].

16. Y. Wang and P. Mukherjee, Phys. Rev. D 76, 103533 (2007) [astro-ph/0703780].

17. A. R. Liddle, P. Mukherjee, D. R. Parkinson, A\&G 47 4.30-4.33 (2006) [astro-ph/0608184].

18. P. Mukherjee, D. R. Parkinson and A. R. Liddle, Astrophys. J. 638 L51-L54 (2006) [astro$\mathrm{ph} / 0508461]$.

19. J. Skilling, http://www.inference.phy.cam.ac.uk/bayesys/. 